\title{
Modernité, Postmodernité Et Impérialisme Occidental
}

\author{
Dr Maurice Gning \\ Université Gaston Berger de Saint-Louis UFR \\ de Lettres \& Sciences Humaines Sénégal
}

Doi: 10.19044/esj.2018.v14n5p386 URL:http://dx.doi.org/10.19044/esj.2018.v14n5p386

\begin{abstract}
The objective of this study is to underscore the close link between modernity and postmodernity, two ideologies that are, quite often, opposed. Based on history, geopolitics, interculturality and the views of thoerists and critics of these thoughts, mainly those of Lyotard, Derrida, Foucautlt and the marxists, this paper attempts to demonstrate that the opposition between the two ideologies is only formal. They have the same purpose, namely to fulfil an imperialist task. If modernity is the thought of colonialist Europe at the time of the industrial revolution, postmodernity is that of neocolonialist America in the age of globalization. Both are carriers of universal chaos.
\end{abstract}

Keywords: Modernity, postmodernity, imperialism, colonialism, neocolonialism

\section{Résumé}

Le but de cette étude est de mettre en lumière le lien étroit entre la modernité et la postmodernité, deux courants de pensée qui sont, assez souvent, opposés. En nous fondant sur l'histoire, la géopolitique, l'interculturalité et les points de vue des théoriciens et critiques de ces pensées, notamment celles de Lyotard, Derrida, Foucault et des marxistes, nous démontrons dans ce travail que l'opposition entre les deux idéologies n'est que formelle. Elles poursuivent la même finalité, à savoir accomplir un dessein impérialiste. Si la modernité est la pensée de l'Europe colonialiste à l'époque de la révolution industrielle, la postmodernité est celle de l'Amérique néocolonialiste à l'heure de la mondialisation. L'une comme l'autre sont porteuses de chaos universel.

Mots-clés : Modernité, postmodernité, impérialisme, colonialisme, néocolonialisme 


\section{Introduction}

Dans cette étude, nous nous intéressons à deux courants de pensée, la modernité et la postmodernité, souvent aussi appelés le modernisme et le postmodernisme. Cependant, les termes «modernisme» et « postmodernisme » font généralement référence aux mouvements artistiques associés respectivement aux courants de pensée moderne et postmoderne. C'est pour éviter la confusion avec les dimensions esthétiques qu'elles impliquent, et surtout pour mettre exclusivement l'accent sur leurs contenus idéologiques, que nous privilégions, ici, l'emploi de «modernité » et de « postmodernité ». La dernière prend son sens dans sa critique de la nature eurocentrique et hégémonique de la première, dont elle débusque les fondements épistémologiques, pour proposer ensuite un tout nouveau mode de vie et de pensée, respectueux de la pluralité des visions du monde. Cela a amené une frange de la critique à opposer ces deux pensées occidentales, aussi bien dans leurs principes que dans leur orientation.

En s'érigeant contre l'impérialisme de la modernité, et en invalidant son discours totalisant, la postmodernité donne l'air d'une idéologie libératrice. Pourtant, à y voir de plus près, l'on se rend compte qu'elle s'inscrit dans la même logique impérialiste que la modernité.

En puisant dans l'histoire, la géopolitique, l'interculturalité et dans les réflexions des théoriciens et critiques de ces deux courants, notamment celles des marxistes et des poststructuralistes comme Lyotard, Derrida et Foucault, ce travail se fixe pour objectif de montrer que ces deux courants idéologiques, analysés sous l'angle de leur finalité, entretiennent une relation très étroite. Ils sont, en effet, deux visions impérialistes qui, compte tenu de certaines réalités historiques, diffèrent seulement par leurs formes. Par ailleurs, il sera question de voir comment le mode de vie que propose la postmodernité constitue un moyen très subtil d'imposer une vision unique, porteuse d'un chaos universel vraisemblablement pire que celui de la modernité.

\section{La modernité hégémonique et ses avatars}

La modernité est cette idéologie occidentale née depuis la Renaissance et qui a supplanté celle du Moyen-âge. Les nombreuses théories et découvertes scientifiques des 15ème et 16ème siècles ont contribué à changer complètement la vision du monde de l'Occident. Désormais, ce n'est plus l'Eglise Catholique avec ses dogmes «scientifiquement erronés » qui doit guider la pensée et l'action humaine, mais plutôt la raison, caractéristique essentielle de l'homme.

Grâce à la lumière de la raison, de ce bon sens dont Descartes disait qu'il est « la chose la mieux partagée au monde », la modernité occidentale a estimé devoir repousser l'obscurantisme et l'ignorance. Elle s'est également donnée à lire dans son refus de se soumettre aveuglement au dogme, à 
l'autorité, pour ainsi promouvoir la liberté de conscience, la connaissance scientifique, seules conditions nécessaires à l'avènement d'un monde juste, équitable, émancipé et maîtrisé ». Ce monde, tel qu'il est articulé par la modernité, est celui dans lequel l'individu, pleinement épanoui, sera réconcilié avec lui-même en tant qu'être universel, «sujet-dans-le-monde se sentant responsable de lui-même et de la société » (Touraine, 2000).

En estimant que l'application rigoureuse de la raison est l'unique voie pour atteindre l'idéal de progrès pour le genre humain, la pensée moderne a érigé sa vision rationaliste en loi universelle. Discours légitimant, elle a du coup discrédité, invalidé puis rejeté toutes les autres visions du monde, car n'étant pas conformes à ses principes rationnels. Celles-ci sont ainsi jugées obscures, primitives, barbares et rétrogrades et les peuples qui en sont porteurs, considérés comme évoluant en marge de l'histoire. A ce propos, le jugement bien célèbre que porte Hegel (2007) sur le continent africain est sans appel :

Ce continent n'est pas intéressant du point de vue de sa propre histoire, mais par le fait que nous voyons l'homme dans un état de barbarie et de sauvagerie qui l'empêche encore de faire partie intégrante de la civilisation. L'Afrique, aussi loin que remonte l'histoire, est restée fermée, sans lien avec le reste du monde ; c'est le pays de l'or, replié sur lui-même, le pays de l'enfance qui, audelà de l'histoire consciente, est enveloppée dans la couleur noire de la nuit.

Penseur de la dialectique de l'histoire, Hegel est l'un des plus illustres théoriciens du courant moderne. Son point de vue sur ce continent traduit parfaitement l'esprit de cette idéologie qui associe l'Afrique aux ténèbres, à l'âge de l'enfance ou de la Tabula rasa, autrement dit, de la virginité de l'esprit humain.

Dans son ambition de réaliser l'universalité des communautés, l'Occident moderne a cru nécessaire d'apporter la lumière de sa raison à tous ces peuples «plongés encore dans l'obscurité de l'ignorance » et qu'il urge d'introduire dans la modernité et l'histoire. Telle est la vision qui justifierait la vaste entreprise coloniale de l'Occident «en faveur» des peuples «barbares» de l'Afrique qui se voient ainsi soumis à l'exercice de « civilisation ».

Assez curieusement, l'Occident qui vient « civiliser » des « barbares » avaient visiblement d'autres préoccupations plus sérieuses. Les pays qui le composent étaient, pour l'essentiel, engagés dans une logique d'extension de leurs territoires dans un contexte de mercantilisme. Cette politique expansionniste a donné naissance aux grands Empires européens des $16^{\text {ème }}$ et $17^{\text {ème }}$ siècles, constitués dans l'Amérique et l'Océan Pacifique. Au 19ème siècle, la plupart de ces pays occidentaux avaient connu la révolution 
industrielle. Industrialisés et surpeuplés, ils avaient besoin de matières premières pour leurs industries, de l'espace pour leurs surplus démographiques, un grand marché pour écouler leurs produits manufacturés. Or, le mouvement révolutionnaire des 13 colonies anglaises en Amérique, qui a abouti à leur l'indépendance en 1783, sous le nom des Etats-Unis d'Amérique, avait précipité l'émancipation des autres colonies de ce continent.

Les colonies américaines étant perdues, les pays colonisateurs se sont tournés vers l'Afrique et l'Asie pour reconstituer leurs empires. C'est la seconde phase de la colonisation dont la motivation, essentiellement économique, est bien résumée par cette célèbre phrase de l'homme d'Etat français, Jules Ferry: «la politique coloniale est fille de la politique industrielle ». C'est dire que la thèse humanitaire et idéologique n'est qu'une belle couverture dans laquelle l'Occident a bien dissimulé son projet impérialiste. En tout état de cause, la « mission civilisatrice » s'est révélée être une atteinte grave à la dignité de l'homme noir, qu'elle a dépouillé de son essence au double plan culturel et économique.

Parallèlement à ce discours rationaliste très souvent associé au capitalisme et qui est à la base de l'assujettissement des peuples d'Afrique et d'Asie, se développent d'autres récits totalisants au rang desquels on note le socialisme et le marxisme-communisme. En tant que discours progressistes, porteurs de projets émancipateurs pour l'humanité, ces métarécits sont des composantes de la grande pensée moderne, même s'ils s'opposent radicalement à son orientation capitaliste.

Au socialisme et au marxisme-communisme se sont adossés d'autres discours qui vont malheureusement vite s'écarter de leurs lignes idéologiques pour se transformer en véritables outils despotiques, dont l'unique but est d'assouvir le dessein funeste de leurs tenants. Aussi ont-ils donné naissance aux pires régimes totalitaires que le monde ait jamais connus, le Stalinisme en URSS et le Nazisme en Allemagne, qui se sont révélés fatals à toute l'humanité.

Foncièrement hégémoniques, violentes et fondées sur des critères d'exclusion de certains individus et couches sociales, ces doctrines ont servi de fondement idéologique à de folles entreprises génocidaires comme l'extermination d'environ 6 millions de Juifs par l'Allemagne Nazi et les goulags dans lesquels le régime totalitaire soviétique aurait fait périr une dizaine de million de personnes, essentiellement des opposants au pouvoir tyrannique de Staline. De plus, la politique expansionniste des ces régimes dictatoriaux, notamment celle de l'Allemagne nazi, a été l'une des causes de la guerre la plus meurtrière de l'histoire humaine, en l'occurrence la Seconde Guerre mondiale, dont le bilan approximatif en termes de perte en vies humaines est estimé à plus de 60 millions d'individus. 
Catastrophes de la colonisation, naissance de régimes totalitaires aux conséquences dramatiques, horreurs de la Première et de la Seconde Guerre mondiale, aggravés par l'utilisation d'armes hautement dévastatrices issues directement du progrès de la science et de la technique, destruction graduelle de l'environnement soumis à une pollution continuelle, guerre froide entre puissances américaine et soviétique, course à la bombe atomique qui met permanemment en danger l'existence sur terre, tel est, en résumé, le lourd bilan de la modernité qui a entaché ses énormes avancées technologiques, inspirant ainsi un sentiment général de profonde déception. C'est ce qui transparait dans ce tableau sombre que dresse Rosenau (1992: 5)

Modernity entered history as a progressive force promising to liberate humankind from ignorance and irrationality, but one can readily wonder whether that promise has been sustained. As we 1 . ./ approach the end of the twentieth century, the <modern> record -

world wars, the rise of Nazism, concentration camps 1.../, genocide, worldwide depression, Hiroshima, Vietnam, Cambodia, the Persian Gulf, and a widening gap between rich and poor 1.. . / makes any belief in the idea of progress or faith in the future seem questionable.

Ainsi que le font remarquer les tenants du marxisme qui pointent un doigt accusateur à l'esprit capitaliste, les hécatombes liées aux guerres et aux génocides des régimes absolus du 20ème siècle ont causé une "coupure qualitative dans la trajectoire historique de la civilisation capitaliste ». Comme par magie, le barbarisme, dans sa pire forme, semble être directement sorti du mode de civilisation que l'Occident moderne imposait aux peuples dits barbares. Theodore Adorno de l'Ecole de Francfort l'aura si bien compris, qui qualifie l'holocauste nazi «d'une barbarie qui s'inscrit dans le principe même de la civilisation $»$.

Dans sa quête de progrès sous la responsabilité et l'éclairage de l'Occident rationnel, l'humanité a abouti à ces événements tragiques qui ont fait perdre à la modernité toute crédibilité. Sa dissolution a entraîné une " rupture épistémologique », selon l'expression de Gaston Bachelard. Il faut donc envisager le monde autrement, repenser le 21ème siècle et les bases mêmes de la civilisation, pour paraphraser Enzo Traverso. Il faut créer de nouvelles idées, car ce sont elles, nous dit Jacqueline Russ, qui gouvernent le monde. Mais l'Occident a-t-il encore le courage et la force de concevoir de nouvelles idéologies qui s'appliqueraient encore à toute l'humanité ? Tout porte à croire qu'il est devenu plus modeste. Il s'est contenté de tirer les conséquences de l'échec de la modernité ; échec à partir duquel il a, pourtant, établi de nouveaux paradigmes, une vision du monde appelée la postmodernité, que Jean-François Lyotard, l'un de ses premiers et plus célèbres théoriciens, définit comme « la condition des sociétés déçues par les 
promesses du modernisme ». Ce nouveau courant de pensée s'inscrit ainsi dans une logique de dénonciation et de rejet des fondements épistémologiques de la modernité.

\section{La critique postmoderne des fondamentaux impérialistes de la modernité}

La postmodernité ne se limite pas à constater l'autodissolution des idéaux du modernisme. Les penseurs postmodernes dissèquent le discours structurant de ce courant idéologique pour en révéler la nature et la finalité. L'une des conclusions majeures qui ressortent de leurs analyses, c'est que la modernité est un projet qui a une visée essentiellement impérialiste. Comme le rappelle Turnbell (2O10: 6), "Postmodernist thinkers argue that rationality had no firm foundations, and was itself merely one narrative among others. Hence, they presented the Enlightenment not as a common project of the advancement of knowledge, but as a vehicle of power". Les postmodernistes considèrent que l'idéologie rationnaliste n'est qu'un récit messianique qui n'a aucun autre fondement en dehors de la volonté inavouée de domination. Pour ces penseurs, le pouvoir qu'implique le discours moderniste est consubstantiel même au postulat de départ de cette idéologie, à savoir le sujet rationnel. Ce sujet autonome, qui fonde son existence sur sa propre conscience, pose en principe que le monde extérieur est objet sur lequel il a l'impérieux devoir d'agir. Les postmodernistes estiment que cette « relation sujet-objet qui est au cœur du modernisme a engendré le rapport dominant-dominé, maîtreesclave » (Kom 37). Cette ambition hégémonique du sujet moderne est d'ailleurs clairement déclinée par l'un de ses pères fondateurs, René Descartes, lorsqu'il enjoint le sujet rationnel d'être «maître et possesseur de la nature $»$.

L'ambitieux projet d'appropriation du monde est d'autant plus envisageable que les modernistes estiment que la raison est capable de saisir la vérité et de fonder une connaissance empirique, objective et universelle. C'est justement ce postulat de base que les postmodernistes remettent totalement en cause. Soutenant que le monde est hétérogène, fragmentaire et changeant, ils rejettent la vision ontologique selon laquelle le sujet rationnel est à même de rendre objectivement compte du réel. Par ailleurs, à la suite de Nietzsche et de Freud, ils nient toute autonomie du sujet qu'ils estiment être sous-tendus par des forces psychiques, des structures qui échappent complètement à son contrôle. Le sujet ne saurait accéder à la réalité des choses, encore moins fonder un savoir proprement objectif et neutre. Toute connaissance devient dès lors relative, rendant entièrement obsolète toute conception totalisante du monde.

Cette critique formulée en l'encontre du sujet moderne est précisément l'œuvre d'intellectuels français de divers horizons disciplinaires et qui sont généralement regroupés sous l'appellation commune de 
«poststructuralistes ». Composés, pour l'essentiel, de Jacques Derrida, Michel Foucault, Jean Baudrillard, Jean-François Lyotard, Jacques Lacan, Roland Barthes et de Gilles Deleuze, ces poststructuralistes ou les tenants de ce que le monde anglo-saxon appelle la « French theory » sont aussi assimilés, à tort ou à raison, au courant postmoderne. Toujours est-il que leurs principes poststructuralistes forment le noyau même de la pensée postmoderne.

En battant en brèche la prétention du sujet rationnel à l'autonomie, la transparence, la discursivité, l'objectivité et à l'universalisme, les poststructuralistes amputent d'abord le discours moderne de son "socle originel », puis le vident de sa substance, enfin, l'invalident. Mieux, ils s'attachent à montrer comment, en érigeant des postulats purement subjectifs en règles universelles, l'épistémologie moderne se révèle être un véhicule de pouvoir. Il est ainsi question pour eux de déconstruire cette idéologie du plus fort. La pensée de Derrida et celle de Foucault s'inscrivent dans cette entreprise de déconstruction.

A l'image de ses pairs poststructuralistes, Derrida part d'un certain nombre de faits qui mettent en mal le fondement même de la pensée moderne. Tout d'abord, il considère qu'il n'y a pas de réalités en dehors du langage. En effet, le langage est par excellence le moyen par lequel le sujet conçoit, interprète et exprime la réalité sur laquelle il prétend avoir prise. «Toute pensée consciente », affirme Nietzsche, «n'est possible qu'avec l'aide du langage » (Marton: 2012, 227). Or, il ressort des analyses de plusieurs penseurs, y compris Nietzsche lui-même, que « dans la mesure où on n'a pas accès à la chose en soi, les mots ne peuvent pas correspondre aux choses ellesmêmes ». Le langage n'étant pas en mesure de traduire convenablement la réalité, les poststructuralistes n'y voient qu'un outil au service de l'expression des intérêts des uns et des autres, d'autant plus qu'il est gouverné par des structures qui transcendent le sujet. Fort de ce principe, Derrida soumet à l'analyse critique la métaphysique occidentale pour déceler les préceptes idéologiques qu'il charrie. C'est pour ensuite se rendre compte que celui-ci est essentiellement logocentrique, c'est-à-dire centré sur le logos ou la raison discursive occidentale.

Cette raison discursive établit un certain nombre de critères à partir desquels elle se définit, se particularise et, par conséquent, se différencie des autres formes de pensée. Très rapidement, l'Occident a posé sa rationalité en vérité absolue, la faisant ainsi passer de l'empirique au normatif, c'est-à-dire "d'une critique des faits vers une critique de ce-que-l'on-doit-faire-étantdonné-les-faits » (Timmermans, $2000: 3$ ). Les penseurs postmodernes font remarquer que ce passage du sujet rationnel de l'empirique au normatif procède d'une volonté de l'Occident d'instaurer un certain ordre dans le monde, après l'avoir "vidé de toute consistance ontologique stable " (Baudrillard, 2012 : 36) et réintroduit dans le chaos originel foucaldien, par le 
biais du cogito cartésien qui l'a arraché à son socle métaphysique et, plus tard, à son assise religieuse.

La mission d'ordonnancement du monde - autrement dit, celle de domination- que s'est assignée la modernité implique de séparer, de distinguer, puis d'hiérarchiser, toujours dans une démarche empirique, les éléments constitutifs de l'existence. Baudrillard (36) l'explicite fort bien :

Il fallut donc à Descartes, pour en arriver au sujet autonome et mesure de toute chose, opérer une sorte de processus de mise en ordre, fondé sur la différenciation, la division, la désunion et l'exclusion. De l'obscurité initiale dans laquelle le doute hyperbolique plonge tout ce qui existe, il faudra donc à Descartes cela dit de manière très schématique - procéder à une distinction entre la matière inorganique et la matière organique ; puis, opérer une seconde série de différentiations entre le végétal, l'animal et l'humain ; une fois rendu à l'humain, il lui faudra effectuer de nouvelles distinctions entre la conscience de l'homme et son corps, qu'il relègue au domaine de la matière, puis en distinguant l'homme qui dort (qui n'est donc pas conscient) et le fou (qui ne sait pas ou plus faire usage de sa raison) de l'homme éveillé, conscient, doté de raison (et qui est donc en mesure de faire l'expérience du Cogito). En tant que logos, le sujet cartésien se trouve à participer de la Raison Divine, et se trouve au plus près de Dieu dans la grande hiérarchie des êtres

Selon Derrida, une telle hiérarchisation traverse de bout à l'autre l'histoire de la philosophie occidentale à travers le primat qu'il accorde à la notion véhiculant et perpétuant le logocentrisme au dépens de celle à laquelle elle s'oppose et qui paradoxalement lui donne sens. Par exemple, dans un texte s'inscrivant dans le registre rationnel, «blanc » et systématiquement valorisé et opposé à 《noir », «centre » à 《périphérie », «civilisation» à « barbarisme ", « riche » à " pauvre ", « ordre » à « désordre », « stable » à « instable », « cohérent» à « incohérent», etc. Derrida considère que cette opposition binaire est fallacieuse, étant donné que l'un ne saurait se concevoir, exister sans l'autre. Par ailleurs, le mot n'étant pas autoréférentiel, son interprétation est ouverte et non figée.

Privilégier une signification au détriment de l'autre, c'est mettre très arbitrairement en avant son point de vue sur le monde. Tel est, dans la vision derridienne, le péché que l'Occident n'a cessé de commettre depuis la nuit des temps et dont il urge de le purifier. C'est cette fin que Derrida assigne à son vaste programme déconstructionniste qui consiste à traquer, dans les mots qui forment l'ossature textuelle de la pensée occidentale, les traces du logos, en vue de le déconstruire, de subvertir la logique hiérarchique qu'il implique, d'en établir l'incohérence, la contradiction, le non-sens auquel il aboutit, et 
toute la subjectivité qui s’y rattache. Il fait donc perdre aux mots leur fixité idéologique, joue sur l'élasticité de leurs significations, en déplaçant continuellement celles-ci à travers des glissements sémantiques. Ce faisant, il leur ouvre la voie à une multitude d'horizons de significations, mettant ainsi en lumière et anéantissant l'imposture du logos. N'ayant pas de capacité référentielle, le logos occidental ne fait que créer sa propre réalité, sa propre subjectivité, tout en s'autoproclamant objectif, car fondé sur une méthode scientifique qui ne saurait se tromper.

Justement pour Foucault, cette vérité scientifique non seulement se trompe, mais est fondamentalement trompeuse. Nourrissant le même scepticisme que Derrida à l'égard du langage quant à sa capacité à exprimer la réalité, il estime que le discours rationaliste du courant moderne n'a aucun fondement objectif. Sa démarche généalogique, qui le rapproche de Nietzsche, permet à Foucault de conclure que chaque époque produit un discours cohérent à partir de l'ensemble des connaissances de la période, qu'il appelle épistémè. Autrement dit, l'époque établit un cadre général dans lequel se déploie le discours normatif qui détermine ce qui doit être dit et ce qui doit être fait. La modernité, en tant que discours idéologique fondé sur la rationalité, constitue un des moments épistémiques de l'Occident.

Les épistémès, soutient-il, sont changeantes, contingentes, aléatoires et subjectives. Elles ont comme unique but de réguler le désordre inhérent à l'existence humaine, d'imposer un ordre. En cela, elles sont un véhicule de pouvoir. Il précise que ce discours normatif émane de la classe dirigeante. En définissant, non sans intérêt égoïste, les contours du savoir, celle-ci impose ses normes et consolide son pouvoir en tant que l'unique détentrice de la vérité à laquelle les autres doivent se conformer. Celui qui détient cette vérité, détient en même temps le pouvoir. Dans Surveiller et Punir, il exprime cette relation dialectique entre le savoir et le pouvoir, à travers cette célèbre formule : « Il n'y a pas de relation de pouvoir sans constitution d'un champ de savoir, ni de savoir qui ne suppose et ne constitue en même temps des relations de pouvoir ». Or, à l'époque moderne, le savoir synonyme de vérité est, comme le dira plus tard Lyotard, immanent au jeu scientifique. C'est la science, dans sa prétendue démarche méthodique et objective qui établit la véracité d'un discours.

A travers le prisme de cette épistémè dont le soubassement est la rationalité, l'Occident a marginalisé puis voué aux gémonies tous les discours sortant de son cadre théorique, perpétrant ainsi une véritable agression culturelle, dont les peuples victimes portent toujours les traces. L'amer constat d'Aimé Césaire (2004), dans Le Discours sur le colonialisme, en donne une idée plus précise:

Moi, je parle des sociétés vidées d'elles-mêmes, de cultures piétinées, d'institutions minées, de terres confisquées, de religions 
assassinées, de magnificences artistiques anéanties, d'extraordinaires possibilités supprimées. On me lance à la tête des faits, des statistiques, des kilomètres de routes, de canaux, de chemins de fer. Moi, je parle de milliers d'hommes sacrifiés au Congo-Océan. Je parle de ceux qui, à l'heure où j'écris, sont en train de creuser à la main le port d'Abidjan. Je parle de millions d'hommes, arrachés à leurs dieux, à leur terre, à leurs habitudes, à leur vie, à la vie, à la danse, à la sagesse.

En analysant les conditions historiques de la naissance des discours idéologiques, Foucault a mis au jour leur essence hégémonique et leur caractère particulièrement arbitraire. Il a également montré comment ce pouvoir, visible à toutes les échelles sociales, partout dans la vie, et s'articulant dans le langage, est sous-tendue et maintenue par une forte architecture institutionnelle qui crée, exclut et domine un certain nombre de groupes dits minoritaires.

A la lumière des théories poststructuralistes, notamment celles derridienne et foucaldienne, qui perpétuent une tradition de remise en cause des valeurs fondatrices de la modernité initiée par les "penseurs du soupçon », Marx, Nietzsche et Freud, nous pouvons dire, avec Habermas, que la raison est « démasquée tout à la fois comme une subjectivité qui assujettit tout en étant elle-même asservie, et comme une volonté de maitrise instrumentale » (Boizette, 2013 :4). Après avoir concrètement révélé ses limites à travers les multiples catastrophes qu'elle a induites, la modernité est déconstruite, sur le plan strictement des idées, est frappée définitivement d'inanité. A son faux universalisme et prétendue objectivité, les postmodernes ont substitué le relativisme, c'est-à-dire une pluralité de subjectivités, de vérités variantes en fonction des multiples points de vue. Ce faisant, ils donnent directement la possibilité aux discours périphériques, jadis invalidés, de mettre en évidence et de faire prévaloir leurs vérités, leurs interprétations de la réalité.

L'un des plus célèbres discours périphériques, qui a repris à son compte l'interprétation de l'histoire, est, sans doute, la théorie postcoloniale. Impulsée, en partie, par le nouveau courant poststructuraliste-postmoderne et nourrie de la pensée déconstructionniste de Frantz Fanon et celle d'Edward Saïd, la théorie postcoloniale s'est particulièrement attaquée à la modernité occidentale qu'elle estime être un discours ethnocentrique, européocentrique et colonialiste. Tout en jetant un regard critique sur le fait colonial et son impact sur les peuples qui en sont victimes, les tenants de la théorie postcoloniale privilégient une relecture lucide de la culture des anciennes colonies à partir des paradigmes qui leur sont propres. L'on constate également l'émergence et l'épanouissement d'autres subjectivités telles que le mouvement féministe et celui des LGBT (lesbiennes, gays, bisexuels et transgenres), jadis exclus aussi des grands récits modernes. La multiplication 
des discours témoigne ainsi de la fin de la vision unitaire de la modernité et du caractère libérateur et hétérogène de la pensée postmoderne qui lui a succédé.

\section{Les principes anti-impérialistes de la postmodernité}

La postmodernité est le courant de pensée dominant de notre époque contemporaine. Compte tenu, entre autres, de la pluralité des domaines auxquels elle s'applique, la postmodernité n'a pas, à ce jour, une définition universellement acceptée. Toutefois, ces principes majeurs sont bien connus. Ceux-ci ont la particularité d'être anti-impérialistes, car récusant tout discours porteur de principe normatif universel. Timmermans (2000:32) le rappelle ainsi :

La pensée «postmoderne » aurait donc pour trait fondamental de ne pas prétendre à l'impossible synthèse entre objet et sujet, entre faits et normes, ou entre connaissance et action. Elle ne chercherait pas à ramener sous une seule loi offerte à notre connaissance des façons de vivre, d'agir, de sentir, profondément différentes.

Ce rejet de toute vision totalisante et le rappel de la pluralité des points de vue trouvent leur assise dans le principe majeur postmoderne selon lequel il n'y a pas de vérités absolues, mais de possibilités infinies d'interprétation de la réalité. Au discours subjectif du centre, il convient d'opposer celui des périphéries qui, comme le rappelle Edward Saïd dans Orientalism, ne sont que des créations de l'Occident. La postmodernité célèbre l'éclatement du centre occidental et inaugure l'ère du décentrement, de la profusion de plusieurs centres aussi valables les uns que les autres, créant ainsi, comme le rappelle Nkolo Foé (2008: 150), un «monde polycentrique ». Il appartient alors à ces peuples, jadis périphériques mais désormais se reconnaissant comme des centres, de réinterpréter et de réécrire leurs réalités falsifiées ou même niées, en mettant en avant leurs vrais éléments culturels, d'évaluer et de déconstruire le discours colonial ou raciste pour que, enfin libérés et réconciliés avec euxmêmes, ils puissent sereinement envisager le futur. N'est-ce pas là le bien fondé de tous les mouvements et discours de libération ou de résistance des peuples longtemps asservis? Nous pouvons en citer les multiples luttes d'indépendance des nations colonisées à travers le monde, les mouvements des droits civiques aux Etats Unis, le discours de la négritude du trio Senghor, Césaire et Damas, la lutte contre l'Apartheid en Afrique de Sud, la théorie et les littératures postcoloniales, l'Afrocentrisme, etc.

La pensée postmoderne n'est pas seulement solidaire des peuples victimes de la domination coloniale, mais aussi de tous les groupes exclus, jugés et condamnés à partir de réalités exogènes. C'est le cas des femmes prises au piège d'un monde phallocratique qui doivent se libérer du joug de la domination masculine; d'où le mouvement féministe, c'est le cas aussi des 
homosexuels à qui elle reconnaît une orientation sexuelle valable et dont elle approuve et soutient la lutte pour la reconnaissance au plan international. Bref, ce courant de pensée accorde une attention particulière à tous les groupes dits minoritaires qui portent encore le poids de la domination quelle que soit la forme qu'elle puisse prendre.

Chaque peuple, chaque groupe a sa culture. Aucune culture ne peut être comprise avec des axiomes qui lui sont étrangers, mais à partir de ses propres modèles théoriques. Le monde est multiculturel, multiconfessionnel, multilingue, culturellement pluriel. La postmodernité va au-delà de la simple constatation de la multiplicité des cultures, du foisonnement des petits récits, pour paraphraser Lyotard. Elle prêche la nécessité de dialogue entre ces ensembles culturels. Cet appel au dialogue des cultures, communément appelé l'interculturalité, serait inévitable pour la coexistence pacifique entre les peuples, entre les religions, entre les ethnies, entre les hommes qui doivent non seulement s'accepter dans leurs différences et particularités, mais s'intégrer. Nous sommes au plein cœur du métissage culturel de Senghor, de la civilisation non pas universelle mais de l'universel, de la rencontre de plusieurs cultures.

Cette civilisation a donné naissance à un homme nouveau, l'homme cosmopolite, le citoyen du monde, qui est à la fois enraciné dans sa culture et ouverte à toutes les autres. Parce qu'il est le produit d'un ensemble de réalités hétérogènes et parfois contradictoires, cet homme nouveau est transculturel, complexe et hybride, comme en témoignent les écrits de Homi Bhabha, l'une des figures de proue du postcolonialisme. L'identité humaine n'est pas une donnée fixe, mais mouvante, dynamique. Elle est fonction de référentiels qui sont très changeants.

Le courant postmoderne accorde une importance toute particulière à cet individu cosmopolite. Il se préoccupe surtout de son bien-être physique, ici sur terre car, comme le dit Nietzsche, il n'y a nul réel au-delà du réel. En l'absence de vérité transcendante, l'homme postmoderne devient la mesure de toutes choses. A chacun sa vérité, dit-on souvent. Vivre, c'est choisir et se faire plaisir; c'est profiter des nombreuses possibilités que le monde hyperculturel et postindustriel offre à tous les nouveaux. Désormais, il est interdit d'interdire. Selon Lipovetsky, " la postmodernité représente le moment historique précis où tous les freins institutionnels qui contrecarraient l'émancipation individuelle s'effritent et disparaissent, donnant lieu à la manifestation de désirs singuliers, de l'accomplissement individuel, de l'estime de soi ». (Foé, 2008 : 29). Cette vision hédoniste et éclectique de la postmodernité fait qu'elle considère l'individu comme un être libre, un micro centre, une subjectivité insaisissable. Voilà pourquoi elle prône une attitude souple à son égard. Il faut lui laisser le soin d'orienter sa vie dans le respect des grands principes de tolérance et de flexibilité. Il convient donc de créer le 
cadre institutionnel adapté pour lui permettre de choisir et de s'épanouir sans entraver le choix de l'autre. Tel est le grand principe postmoderne qui soustend la plupart des institutions internationales et locales. Ce sont des institutions qui commandent la souplesse d'esprit, qui mettent l'épanouissement de l'individu au cœur de ses préoccupations, un épanouissement qui passe par un choix sans contrainte.

A titre d'exemple, les grandes réformes de l'enseignement supérieur en cours dans beaucoup d'universités africaines, qui sont importées de l'Occident, sont dictées par cette pensée postmoderne. Le LMD, tel que conçu, n'impose rien à l'étudiant en terme de parcours. C'est à lui de choisir, de construire son profil à partir de la masse de formation offerte. Ne dit-on pas souvent que c'est un système souple qui favorise la mobilité des étudiants ? En principe, l'étudiant peut se mouvoir, au plan interne et externe, dans ce système.

Il en va de même des reformes pédagogiques inhérentes au système. L'approche cours qui permettait à l'enseignant d'être " maître et possesseur » de son cours est caduque. Nous sommes à l'heure de l'approche par compétence, souvent appelée approche programme qui exige que l'institut définisse son programme de formation et que les enseignants travaillent de façon collégiale dans un contexte postmoderne de multidisciplinarité, d'interdisciplinarité et de transdisplinarité qui brouille les frontières entre les champs de connaissance. Ce système pédagogique en cours dans beaucoup de pays a décentré l'enseignant. Il n'est plus le «monsieur qui sait tout » et dont les apprenants doivent passivement tout attendre. Il est réduit à un statut de guide. La parole est désormais donnée à ceux qui étaient jadis à la périphérie, ceux qui subissaient la loi de l'enseignant, c'est-à-dire les apprenants. Au-delà du système éducatif, le principe postmoderne de subversion des rapports de force est perceptible dans bien des domaines dans notre société contemporaine.

Au nom de la relativité des choses, au nom de l'urgence du dialogue culturel, au nom de la liberté de choisir, la postmodernité prêche la tolérance, le respect et l'ouverture aux autres. Elle refuse l'esprit dogmatique, les jugements de valeurs et le repli identitaire. Au plan étatique, elle prône la démocratie, la liberté d'expression et de déplacement, et condamne toute tentative dictatoriale, toute atteinte à la dignité et à l'intégrité humaine. Nous sommes à l'ère des droits de l'homme, des droits des enfants, des droits de la femme, et des autres groupes vulnérables, du droit de l'environnement, de l'apprenant entre autres. Ces droits attribués pêle-mêle, et souvent avec beaucoup de générosité, devraient pousser à réfléchir davantage sur la finalité de la postmodernité, surtout si on sait « qu'il n'y a pas de libération sans une nouvelle forme de dépendance » (Charles, $2006: 4$ ) 


\section{Les non-dits de l'anti-impérialisme postmoderne}

Dans son rejet systématique des certitudes occidentales de la modernité, le courant postmoderne se révèle, a priori, porteur d'un projet libérateur pour les peuples et groupes opprimés. A travers ses nombreuses théories déconstructionnistes, il met à la disposition de tous les groupes dominés des outils analytiques capables de remettre en question le centre épistémologique, la pensée impérialiste de l'Occident et de les rétablir dans leur dignité bafouée.

Si des figures emblématiques, la plupart formées à l'école du colon, ont su, en partie, se servir de cette arme intellectuelle pour porter le combat de la défense des anciennes colonies contre l'hégémonie étrangère, force est de constater, aujourd'hui, que l'ennemi est toujours présent. Il a bien établi son pouvoir. Cet ennemi est ressorti du camp des opprimés, de ceux sur qui reposait l'espoir de libération, comme dirait l'homme de culture Kenyan, Ngugi wa Thiong'o. A l'image de l'espoir suscité par le «soleil des indépendances », qui rapidement a cédé la place aux ténèbres du désenchantement, la fin de l'idéologie rationaliste étouffante est loin d'être synonyme de libération pour les peuples qui en ont vécu les affres. En effet, aussi étrange que cela puisse être, la postmodernité, qui a célébré la mort de la modernité et fait miroiter l'espoir de la fin de l'oppression, de la dictature intellectuelle, de l'esprit dogmatique et hégémonique, incarne, peut-être mieux que le courant moderne ravageur, tous ces maux qu'elle dissèque et dénonce. C'est dire à quel point elle est paradoxale et ambiguë, d'où d'ailleurs l'impasse à laquelle toute tentative de la définir aboutit souvent. Le paradoxe insurmontable de la pensée postmoderne, qui lui ôte tout statut de pensée libératrice, se lit à travers ses grands postulats.

Au sujet des principes contradictoires de cette pensée contemporaine, nous pouvons évoquer celui relatif à l'idée assez répandue que « la disparition des grands systèmes signifie que le postmodernisme n'est ni une école de pensée, ni une idéologie - puisqu'il fait la critique des idéologies ». (Lafaye 4). De prime abord, et si on se réfère au contexte de sa naissance et aux réflexions des poststructuralistes qui en ont jeté les bases, la postmodernité « ne défend aucune valeur particulière ». Elle récuse toute valeur essentialiste, à laquelle elle préfère la relativité, l'hétérogénéité, la multiplicité des points de vue. Elle admet la validité des autres discours et, en même temps, les relativise. Ils sont à la fois vrais et faux; par conséquent, ils ne sauraient se prévaloir d'une quelconque valeur légitimante. Sous ce rapport, le courant postmoderne évacue l'essence des autres discours idéologiques, plus qu'il n'en reconnaît la légitimité. La religion, par nature dogmatique, n'échappe pas à l'œil relativiste de cette pensée. Les dogmes religieux sont ainsi réduits à de simples petits récits ou microrécits au même titre que toute autre idéologie, donc n'ayant de sens et de valeurs que pour ceux qui s'y attachent librement. 
Toutefois, à l'image du sujet cartésien qui doute de tout sauf de ce qui le fait douter, le relativisme postmoderne relativise tout, sauf son propre relativisme. Le postmodernisme «impose le relativisme à tous (...) mais jamais il ne relativise son propre credo ", dit Makri (2013). Il pose son relativisme comme une sorte de vérité première, exactement comme le cogito, une vérité indubitable, une valeur normative à partir de laquelle il fonde une nouvelle vision du monde, une sorte de nihilisme qui, fondamentalement, considère comme vide et fausse toute autre considération, en dehors de celles qu'il a lui-même établies.

Plus qu'une idéologie, la pensée postmoderne peut être considérée comme la nouvelle épistémè contemporaine. Makri (2013) va plus loin en la qualifiant de dogme et évoque, avec une pointe d'ironie, le crédo de la foi postmoderne. Nous voici donc à la case de départ, à l'éternel recommencement nietzschéen : l'Occident, culturellement vide et désenchanté, mais fort de ses nouveaux principes postmodernes et de sa technologie super sophistiquée face à un « tiers monde » culturellement, économiquement et politiquement réduit à sa plus simple expression.

Une nouvelle page de domination s'ouvre ainsi, mais dans un contexte bien différent de celui dans lequel le discours impérialiste du modernisme a vu le jour et s'est propagé. Nous sommes à la fin de la Deuxième Guerre mondiale, à la fin de la Guerre froide, marquant la fin du monde bipolaire et l'établissement d'un Nouvel Ordre mondial polarisé autour d'une seule et unique puissance: les Etats-Unis d'Amérique. En effet, sortis doublement victorieux de la Deuxième Guerre mondiale et de son combat idéologique contre l'Union Soviétique, son plus grand rival, les Etats-Unis sont devenus, comme l'écrivait Zbigniew Brzezinski, «la première puissance mondiale réelle ». La vie étant un perpétuel rapport de forces, cela va de soi que l'Amérique s'attache, dans ce contexte qui lui est entièrement favorable, à conforter et à maintenir son leadership international.

Chantre de la démocratie, ancienne colonie anglaise ayant acquis son autonomie au prix d'une guerre d'indépendance, engagée dans la lutte contre l'oppression, comme le prouve le rôle stratégique qu'elle à joué dans le vaste mouvement de décolonisation et la chute des grands empires européens, un des pays fondateurs de l'ONU dont l'objectif majeur affiché est de préserver la paix mondiale, l'Amérique ne peut donc pas se lancer dans une nouvelle conquête coloniale ouverte. Il s'agira alors, pour elle, au nom de la sauvegarde de sa suprématie, d'imposer tactiquement son idéologie capitaliste au reste du monde avec la complicité de ces collaborateurs occidentaux, notamment le Royaume-Uni, la France et l'Allemagne.

Doctrine libérale fondée sur la propriété privée et le libre marché, le capitalisme implique un mode de vie basé sur la recherche de l'intérêt personnel qui a pour corollaire l'individualisme. Ce mode de vie est porté par 
le libéralisme socio-culturel, courant de pensée centré sur l'individu qu'il cherche à affranchir de certaines tutelles dogmatiques comme la religion, et à qui il reconnaît et garantit des droits inaliénables, telles que la propreté et la liberté. Une telle pensée, qui s'oppose à l'emprise de l'autorité et promeut la liberté et l'individualisme, ne pouvait espérer mieux prospérer que dans un contexte où les valeurs traditionnelles de la modernité sont tombées en désuétude et avec elle toutes les institutions régulatrices de la vie sociale. La fin des métarécits, le vide idéologique qu'elle entraîne et le relativisme ambiant de la période donnent un nouvel élan au libéralisme américain, désormais appelé néolibéralisme, que Juignet Patrick (2015) définit comme suit :

De nos jours, le libéralisme a pris une forme différente que l'on peut nommer néolibéralisme. Cette doctrine prône une réduction maximale du rôle de l'Etat associé au développement du marché dans tous les domaines. Sur le plan socioculturel, c'est une idéologie individualiste et hédoniste qui vise l'augmentation des droits individuels et la libéralisation permanente des mœurs. Elle valorise l'intérêt égoïste au détriment du devoir collectif et des valeurs communes. Il s'ensuit un dédain pour les valeurs traditionnelles qui engendre une transformation des mœurs et du lien social. D'une philosophie globale et pondérée, on est passé à une idéologie qui accentue certains traits du libéralisme, en gomme d'autres. Nous qualifions cette nouvelle forme de néolibérale, pour la différencier de la philosophie classique qui restait assise sur des valeurs traditionnelles.

Ce qui est intéressant dans cette définition du néolibéralisme, c'est qu'elle reprend exactement les données fondamentales de la postmodernité que nous avons décrites plus haut. Cela n'est toutefois pas surprenant, si on sait que l'Amérique a été le laboratoire de la pensée postmoderne, en accueillant et en promouvant dans ses campus universitaires, au tournant des années 80, la plupart des penseurs poststructuralistes français, tels que Foucault, Deleuze, Derrida, entre autres, dont elle a rendu célèbres les théories déconstructionnistes, la fameuse French theory, alors qu'elles étaient presque méconnues en France.

La postmodernité serait alors un prête-nom du néolibéralisme. Cette stratégie de nomination permettrait à l'Amérique, sous prétexte de promouvoir les principes de liberté, de pluralité, de tolérance et d'ouverture, subjectivement tirés des théories déconstructionnistes, d'imposer et d'universaliser sa vision néolibérale à une humanité qui porte encore le deuil de la mort des repères idéologiques. Dans cette perspective, bon nombre d'observateurs voient la postmodernité comme une «imposture », un «mensonge intellectuel», une "post-vérité» ou encore un "discours de 
propagande ». Tomislav Sunic se fait plus précis en considérant la postmodernité comme étant l'Amérique et l'américanisme, c'est-à-dire la promotion de l'Homo americanus ou ce qu'il est convenu d'appeler l'Amercian way of life qu'il estime aussi être le système idéologique américain fondé sur un vérité unique. La postmodernité n'est donc pas très différente de la modernité. «L'étape postmoderne aura eu pour effet de nous libérer des grands discours tout en nous réconciliant avec les principes modernes sans pour autant se faire d'illusion à leur propos, elle n'aura en rien aboli la modernité » (Charles, 2006: 5). Pour le philosophe américain, Frederic Jameson, la modernité et la postmodernité ne sont que des formations culturelles qui accompagnent le capitalisme dans ses différentes étapes. La postmodernité, pour lui, est l'esprit de ce qu'il appelle « le capitalisme tardif ou multinational ou de consommation» qui caractérise la société postindustrielle dominée par le consumérisme et le marketing. Elle est ainsi l'accomplissement et le prolongement de la modernité, sous une forme plus sophistiquée. Nous comprenons dès lors le foisonnement de jargons comme " néo-modernité », « modernité hypertrophié » ou « hypermodernité », " ultra-modernité », « sur-modernité », généralement associés à la postmodernité.

Alors que l'idéologie moderne s'était imposée, hier, aux pays de la «périphérie » par l'entremise de la colonisation, celle postmoderne se fait accepter, aujourd'hui, dans ces mêmes contrées, avec la complicité de ses thuriféraires de tous bords, mais surtout grâce à l'ingéniosité des NTIC, qui ont fait sauter, virtuellement du moins, les frontières entre les nations et réussi la prouesse de réduire le monde à un village planétaire. Nous sommes bien à l'ère de la mondialisation, le temps des échanges économiques et culturels entre les différentes nations ; lesquels échanges sont disproportionnés, compte tenu de l'inégalité des forces technologiques, politiques et économiques. Inutile de dire qu'ils sont très largement dominés par les pays développés. Mais, s'il fallait tenir compte de la marchandise que l'Occident, l'Amérique spécialement, exporte le plus et de façon gratuite, dans les « pays du Sud » et même partout dans le monde, la culture postmoderne occuperait certainement la première place.

Il n'est que de voir la manière dont ce courant de pensée façonne les sociétés contemporaines, " oriente désormais graduellement les modalités de l'action dans un monde complexe et reflète de plus en plus les formes quotidiennes de la socialité » (Segin, 2012 :6), pour se rendre à l'évidence que la postmodernité est la caractéristique principale de notre contemporanéité. Elle est perceptible dans le quotidien des hommes et guide la recherche scientifique dans tous les domaines, en médecine, sociologie, éducation, linguistique, anthropologie, philosophie, marketing, mathématiques, littérature, pour ne citer que ceux-ci. «Peut-on échapper à la 
postmodernité ? ", s'interroge Maria Delaperrière (2011) qui constate qu'elle a bien infiltré le paysage culturel de l'Europe de l'Est, ancien bastion du communisme et longtemps à l'abri de ce phénomène culturel occidental.

L'extension de ce courant de pensée à l'échelle planétaire témoigne moins de la capacité d'adaptation des peuples qui l'ont épousé que de la perspicacité et de la subtilité avec lesquelles la bourgeoisie américaine, grâce à la conjugaison de circonstances historiques, a su insérer cette vision du monde dans des cadres conceptuels comme "postmodernité » et «mondialisation». Ces deux paradigmes sont difficilement séparables car l'un implique l'autre. La relation dialectique entre eux est, indirectement, mise en évidence dans ces propos de Nkolo Foe (2008 : 29):

À la faveur du phénomène de grande portée non seulement économique et politique, mais aussi historique et culturelle que constitue la mondialisation, et afin de donner un nom à l'époque actuelle, certaines tendances de la pensée bourgeoise ont ajouté un segment supplémentaire à l'histoire universelle. C'est ainsi qu'après la préhistoire et l'histoire, le pré-moderne et le moderne, l'humanité serait entrée dans un nouveau cycle historico-culturel, celui de la posthistoire ou, plus généralement, de la postmodernité.

Que la postmodernité soit l'idéologie de la mondialisation ou que la mondialisation soit le tremplin de la postmodernité est, certes, une question importante. En revanche, ce qui nous paraît plus essentiel est de voir comment, à travers leur apparence neutre, comme l'ont été les termes « modernisme » et " universalisme » en leurs temps, les concepts «postmodernité » et «mondialisation» effacent tout particularisme, toute subjectivité, toute provenance, et donnent l'air d'appartenir à l'humanité, au monde bouleversé par l'essor de la technoscience. Cependant, autant la raison instrumentale du modernisme-universalisme a été finalement démasquée et dénoncée, autant la finalité du relativisme du postmodernisme-mondialisation est débusquée. Le couple postmodernisme et mondialisation est, aujourd'hui, assimilé au " capitalisme globalisé », au règne sans partage de l'Amérique capitaliste, qui, tout en distillant intelligemment son mode de pensée et de vie à travers le monde, réussit à contenir subtilement toute pensée dissidente. C'est là où réside la pertinence du concept de «totalitarisme mou » que Sunic emploie pour donner un nom à cette stratégie américaine.

Le mal du courant moderne est, semble-t-il, extirpé de la planète, comme l'atteste la reconnaissance officielle de l'autonomie des pays qu'il avait réduits à la colonisation. Celui de son «petit frère-ennemi », le courant postmoderne, semble avoir de beaux jours devant lui. Nous ne voyons pas comment l'humanité pourrait facilement s'extraire de la pensée unique américaine qui se dissimule dans la postmodernité. Avançant à visage masqué, l'idéologie néolibérale américaine est déjà bien répandue dans toutes les 
contrées du monde, et est soutenue par une puissante architecture institutionnelle dictant la marche de l'histoire contemporaine. Nous ne pouvons donc que donner du crédit à la thèse de « la fin de l'histoire » du philosophe et économiste américain, Francis Fukuyama. Par ce terme, il faut entendre la victoire définitive de la démocratie libérale américaine, consacrée par la chute du mur de Berlin, et qui met fin au combat des idéologies ayant toujours rythmé l'évolution de l'humanité.

«Mission terminée », pourrait-on dire pour la postmodernité. Elle a joué un tour à l'histoire qui se répète. Elle a bien parachevé le projet de domination de la modernité en feignant de la critiquer. Elle s'est fait partenaire et porte parole des sans voix, des pays opprimés, avant qu'ils ne découvrent son vrai visage impérialiste. Mais, comme c'est souvent le cas, on se rend compte du mal que lorsqu'il est déjà profond. Les remèdes préconisés, aujourd'hui, par la pensée postcoloniale, l'Afrocentrisme, la multitude de personnalités indépendantes évoluant dans tous les domaines de la vie, se révèlent, pour le moment, inefficaces. Les dominés et tous ceux qui sont acquis à leur cause sont encore trop faibles pour contenir ce géant à la fois visible et invisible, mais, au moins, connu et rebaptisé de son vrai nom : néocolonialisme.

La postmodernité est l'idéologie du néocolonialisme qu'elle accompagne par des manœuvres subtiles. Elle « accentue l'asservissement et les buts du capitalisme » (Yves 2015). C'est pourquoi, à l'enthousiasme qu'elle avait suscité au sein des anciennes colonies a vite succédé un sentiment de désespoir. Ambroise Kom (1997 : 42) fait part de cette grande déception:

En réalité, le triomphe du postmodernisme serait un évènement on ne peut plus ironique. Car, il permettrait de réhabiliter des civilisations et des cultures que l'Occident, aujourd'hui promoteur du postmodernisme, avait lui-même invalidées. Raison pour laquelle les peuples du continent avaient massivement adhéré à la nouvelle civilisation dans l'espoir qu'elle allait les sortir de L'ornière. Or aujourd'hui, tout indique que le doute s'est durablement installé dans les esprits. Le nouveau système éducatif, social, politique et économique a plongé le continent africain dans un inédit désordre social, politique et économique et a engendré un cacabant chaos culturel.

Si le courant postmoderne affecte tant les institutions africaines au point de créer un désordre total, c'est parce que son orientation est clairement inconciliable avec la culture africaine qu'il a fini de phagocyter. Premièrement, c'est une pensée totalement étrangère à l'Afrique, en raison de son cadre spatio-temporel de naissance. Lyotard précise bien, dès l'introduction de son essai, La condition postmoderne (1979), livre qui signe l'acte inaugural de la pensée postmoderne, que son « étude a pour objet la 
condition du savoir dans les sociétés les plus développés ». Deuxièmement, elle est née dans un contexte où ces "sociétés les plus développées", traversaient une réelle crise de sens, un contexte d'épuisement des valeurs occidentales qui, somme toute, est une nouvelle libération.

La postmodernité est justement porteuse de cette culture nihiliste qui célèbre la mort des valeurs classiques, des dogmes, des fausses idoles nietzschéennes et dans laquelle l'homme devient sa propre norme. En l'absence de repères axiologiques, de valeurs normatives, l'interdit perd son sens. Tout devient permis, y compris les passions les plus irréalistes. L'homosexualité, la quête effrénée de profit, de l'argent, de plaisirs de toute sorte, le désir de paraître, le narcissisme, etc., font partie intégrante du nouveau paysage culturel que dessine la postmodernité.

Or, malgré la longue présence occidentale sur le sol africain et les préjudices culturels qu'elle a engendrés, le continent ne s'était pas totalement débarrassé de ses valeurs traditionnelles. Celles-ci sont mêlées aux nouvelles valeurs occidentales, pour donner naissance à une nouvelle culture hybride dans laquelle baigne l'Africain postcolonial.

C'est à cet Africain à moitié modernisé que l'Occident revient encore et propose le nouveau mode vie postmoderne décrit ci-dessus. La plupart des principes de cette nouvelle civilisation ont déjà pris forme en Afrique. Cela est perceptible notamment chez les jeunes qui, friands de nouveautés, raffolent de tout ce qu'offrent le marché mondialisé et les NTIC, vecteurs premiers de la culture postmoderne. La consommation de masse prend de l'ampleur. Les liens familiaux et la solidarité, socles de la société africaine, cèdent graduellement la place à l'individualisme. L'esprit de compétition sociale se fait davantage marquant. L'autorité parentale, jadis très respectée, perd de plus en plus le contrôle des jeunes qui jouissent d'une certaine liberté, et orientent leurs vies selon des modèles de leur choix. Seule la « dérive » sexuelle de la postmodernité, l'homosexualité, désormais reconnue par l'Organisation Mondiale de la Santé comme une orientation sexuelle normale, ne trouve pas encore un écho favorable en Afrique. Voici ce qu'en écrit Houria Bouteldja (Nunes, $2017: 6$ ) :

Il serait temps, une bonne fois pour toute, de comprendre que l'impérialisme - sous toutes ses formes - ensauvage l'indigène : à l'internationale gay, les sociétés du sud répondent par une sécrétion de haine contre les homosexuels. De plus, l'universalité de l'homosexualité est niée : cette notion occidentale ne serait pas adaptée au monde arabe et africain. 
La pression ${ }^{30}$ qu'exerce l'Amérique, principale promotrice de la postmodernité, sur les pays africains pour qu'ils dépénalisent l'homosexualité n'a pas encore produit de fruits. Néanmoins, la bataille contre cette pratique et sa dépénalisation est loin d'être gagnée en Afrique. Mieux, le risque pour le continent de la perdre est réel. Déjà en Afrique de sud, la constitution protège les minorités sexuelles. Au Sénégal, comme d'ailleurs dans plusieurs pays du continent, les homosexuels, appelés dans la langue vernaculaire du pays " goordjigeen », commencent à sortir de l'anonymat, organisent discrètement leurs cérémonies et n'hésitent pas parfois à s'afficher publiquement. Aucune mesure politique conséquente n'est encore prise pour appréhender l'ampleur du phénomène et essayer de le contenir.

La différence culturelle qui séparait l'Afrique et l'Occident s'amenuise à mesure que la postmodernité étend ses tentacules. La pression étrangère, les masses média et la complicité des décideurs politiques africains, dont l'écrasante majorité ont visiblement les mains liées, jouent un rôle essentiel dans cet impérialisme culturel. Les principes postmodernes de pluralité, de diversité, et de dialogue des cultures, tant chantés, révèlent toute leur vacuité sémantique et leur imposture. Deux forces nettement inégales ne peuvent logiquement pas dialoguer. Les règles du jeu sont toujours déterminées par le plus fort, mais jamais en sa défaveur. Le "troisième espace» de Homi Bhabha, c'est-à-dire l'espace hybride et de dialogue, perd son hétérogénéité et devient un espace homogénéisé, occidentalisé. Nous assistons aujourd'hui, dans les anciennes colonies, abusivement rebaptisés " pays partenaires ", à ce qu'il est convenu d'appeler un véritable "génocide culturel». Or, comme l'affirme Marcel Gonçalves (1983 :387) « si une culture en 'digère' une autre, non seulement il y a destruction d'éléments culturels et même un système culturel, mais on tue jusqu'à l'âme d'un peuple, on pratique une certaine forme d'éthnocide».

\section{Conclusion}

La modernité et la postmodernité sont deux courants de pensée dont on a très souvent opposé les principes et orientations. Cela est surtout lié au fait que la postmodernité, née des cendres de l'échec des promesses émancipatrices de la modernité, a affiché, dès le départ, sa farouche opposition aux préceptes modernes comme la rationalité et l'universalisme dont il a démantelé le bien-fondé et dévoilé la visée impérialiste, en s'inspirant,

\footnotetext{
${ }^{30}$ En visite officielle au Sénégal en Juin 2012, le président des Etats-Unis, Obama, demandait à son homologue Sénégalais, Macky Sall, de dépénaliser l'homosexualité, demande à laquelle ce dernier n'a pas accédé, car, disait-il en substance, c'est contraire aux croyances et traditions du pays. En Juillet 2015, ce même Obama, à Addis Abeba, devant l'Union Africaine, s'offusquait de la pénalisation de l'homosexualité en Afrique.
} 
notamment, des travaux des penseurs de la différence ou déconstructionnistes, autrement appelés les poststructuralistes.

Par ailleurs, en se montrant «allergique » à toute entreprise de domination, à toute considération ethnocentrique, et en étant solidaire des peuples et groupes asservis, la postmodernité a, d'emblée, sonné l'heure de la fin de l'oppression. Elle a encouragé et accompagné l'émergence des minorités ethniques et culturelles auxquelles elle a donné la voix à travers le monde, sans oublier les anciennes colonies nouvellement indépendantes.

En dépit de son engagement affiché à lutter contre l'injustice de l'eurocentrisme et à promouvoir la pluralité des voix, pour l'avènement d'un monde juste ayant pour leitmotiv la paix et le développement qui passent par le dialogue des cultures, la postmodernité, n'a pas réussi, loin s'en faut, à juguler l'injustice, le mal de l'impérialisme. S'il en est ainsi, c'est parce qu'elle-même incarne, dans sa forme la plus parfaite, cette hégémonie qu'elle prétend critiquer.

En effet, elle est la nouvelle vision libérale des Etats-Unis d'Amérique qui, à la faveur de circonstances historiques combinées, se sont non seulement hissés au rang de première puissance mondiale, mais sont obligés de changer les règles du jeu pour maintenir leur hégémonie mondiale, face à de potentielles concurrentes comme la Chine et l'URSS. Ils ont donc dissimulé leur politique néolibérale dans des appellations vraisemblablement neutres telles que « postmodernité » et « mondialisation », les deux faces d'une même médaille, dont les principes de base sont exactement ceux du néolibéralisme, avec comme maitres-mots « la liberté de choisir, dans un monde à la fois pluriel et globalisé ». Au fond, la postmodernité ne diffère pas de la modernité, dont elle parachève le projet de domination par des manœuvres très subtiles et raffinées.

La modernité, c'est l'Europe industrialisée et colonialiste qui va à l'assaut du monde, suite aux découvertes scientifiques et techniques de la Renaissance. La postmodernité, c'est l'Amérique postindustrielle, néocolonialiste, patronne des institutions mondiales et accompagnée de ses partenaires occidentaux, qui envahit le monde contemporain, après sa victoire historique contre le communisme soviétique, matérialisée par la chute du mur de Berlin en 1989.

La vieille vision que l'homme occidental se fait de lui en tant que centre et maître du monde n'a jamais changé. De l'esclavage au néocolonialisme en passant par la colonisation, c'est le même dogme qui prévaut. L'Occident réussit à perpétrer et à nourrir la même idée, avec des formes renouvelées au gré des circonstances, et un ensemble de noms et de principes fabriqués de toutes pièces pour mieux la maquiller, au nom de son « devoir » impérialiste envers les autres. Il fait, défait et refait l'histoire. Cette histoire en marche semble prendre fin avec le système néolibéral savamment 
et durablement inscrit dans la culture mondiale, au grand dam des néo colonisés d'aujourd'hui, toujours victimes de l'histoire. Si la modernité a instauré le désordre en Afrique et dans tous les pays dominés à travers monde, la postmodernité a érigé ce désordre en mode de vie, installant ainsi une incertitude généralisée et un profond « malaise dans la civilisation ».

\section{References:}

1. Allard-Poesi, F. et Perret V. (1998). «Le postmodernisme nous propose t-il un projet de connaissance ? » in Cahier, num 263, Mai.

2. Atangana. J. (1973). Chemins d'Afrique, Coll. «Point de vue», Yaoundé, Clé.

3. Balutet, N. (2014). «L'identité au temps de la postmodernité : de l'usage du concept d'hybridité » http://teteschercheuses.hypotheses.org/1141, consulté le 17 décembre 2017.

4. Baudrillard, M. (2012). La notion du sujet chez les poststructuralistes et sa mise en scène dans une série photographique, Thèse soutenue à l'université du Québec à Montréal, septembre.

5. Beti, M. (1957). Mission terminee. Paris: Buchet/Chastel.

6. Boizette, P. (2013). «Introduction à la théorie postcoloniale », http://www.revue-silene.com/images/30/extrait_174.pdf, consulté le 20 décembre 2017.

7. Boisvert, Y. (1995). Le postmodernisme. Montréal : Éditions du Boréal.

8. Boltanski, L. et Chiapello, E. (1999). Le nouvel esprit du capitalisme. Gallimard : NRF Essais.

9. Brune, F. (2003). De l'idéologie aujourd'hui. Paris : éditions Parangon.

10. Césaire, A. (2004). Discours sur le colonialisme. Paris : Présence africaine.

11. Charles, S. (2006). «De la postmodernité à l'hypermodernité » in Philosophie, Revue d'Idées, vol 8, num 1.

12. Coulibaly, L. (1983) L'Autorité dans l'Afrique traditionnelle. Dakar: NEA.

13. Delaperrière, M. (2011). «Peut-on échapper à la postmodernité » in Sociétés. 2 num 112.

14. Fanon, F. (1952). Peau noire, masques blancs. Paris: Seuil.

15. ......... (1960). Les Damnés de la terre. Paris: Maspero.

16. Foé, N. (2008). Le Postmodernisme et le nouvel esprit du capitalisme sur une philosophie globale d'Empire. Dakar : CODESRIA.

17. Gonçalves, M. (1983). «Angola, métissage culturel » in Spiritus, num 93. 
18. Habermas, J. (1988). Les discours philosophiques de la modernité. Paris : Gallimard.

19. Hegel, F. (2007). «La raison dans l'histoire ». (httt://www.mondediplomatique.fr/2007/11/HEGEL/15275), consulté le 27 décembre 2017.

20. Kom, A. (1997). "Culture africaine et enjeux du postmodernisme ». file:///Users/macbook/Downloads/27756-1-28442-1-10$20100706 \% 20$ (1).pdf, consulté le 15 décembre 2017.

21. Lafaye, C. G. «Esthétique de la postmodernité » in Centre Normes, Société, Philosophies http://nosophi.univ-paris1.fr, consulté le 29 novembre 2017.

22. Lyotard, J. F. (1993). Le postmodernisme expliqué aux enfants. Paris : Editions livre de poche.

23. ...............(1979). La condition postmoderne, rapport sur le savoir. Paris : Edition de Minuit.

24. Marton, S. (2012). «Le problème du langage chez Nietzsche : La critique en tant que création " in Revue de métaphysique et de la morale. Presses Universitaires de France, 2, num 74.

25. Makri, Y. (2013) «Islam et postmodernisme» https://www.cilecenter.org/fr/articles-essays/islam-etpostmodernisme-la-question-incontournable-du-sens/, consulté le 15 décembre 2017.

26. Nunes, A. (2017). « Le postmodernisme ne casse pas des briques » in Négatif, bulletin irrégulier-Juillet, num 24.

27. Pisani, E. (1996). «Quelques réflexions pour une critique de la potsmodernité » in Aspects sociologiques, vol 4, 1-2, mai.

28. Segin, T. (2012). Le Postmodernisme une utopie moderne. Paris: l'Harmattan.

29. Timmermans, B. (2000). «Postmodernisme et postcriticisme» in Réseaux, ULB, vol 88-89-90.

30. Touraine, A. (2000). Crítica de la modernidad. Mexico : FCE.

31. Turnbull, Nick. (2010). "Postmodern and Rationality" in Association Revue internationale de philosophie Jan. num 251.

32. Yves (2015). «Le postmodernisme, nouvel âge de l'obscurantisme » http : //.www/cntaittoulouse.l'autre.net/spip.php ?article672, consulté le 07 janvier 2018. 